\title{
Cushing's disease: the desmopressin test as a compelling predictive marker of long-term remission after transsphenoidal
} surgery

\author{
D A Vassiliadi and S Tsagarakis
}

Department of Endocrinology, Diabetes and Metabolism, Evangelismos Hospital, Athens, Greece

\begin{abstract}
Transsphenoidal surgery (TSS) is the treatment of choice in Cushing's disease. However, recurrence rates are substantial and currently there are no robust predictors of late prognosis. As accumulating evidence challenge the accuracy of the traditionally used early postoperative cortisol values, alternative tests are required. The study of Cambos et al., published in a recent issue of the European Journal of Endocrinology, adds to the existing data that support a role of the desmopressin test as an early and reliable predictive marker in successfully TSS-treated patients. However, despite these promising data, the use of this test is hampered by the fact that it can be applied only in patients with a documented preoperative positive test. Moreover, the lack of robust criteria to define positive postoperative responses represents another major limitation.
\end{abstract}

Cushing's disease (CD) remains a disease with high morbidity and mortality (1), due to the harmful effects of cortisol excess which is usually long-standing not only because of delayed diagnosis and treatment difficulties, but also because of substantial rates of recurrence even after successful surgery. Transsphenoidal surgery (TSS) is the treatment of choice; it achieves the most favourable outcome (1) but with an immediate reported success rate varying between centres from 42.0 to $96.6 \%$ (2). The already compromised TSS outcome is further compounded by relapses that may occur even 10 years or more after surgery, ultimately resulting in long-term control of approximately $60-65 \%$ of patients. This means that even patients initially considered to be in remission should be kept on regular and long-term follow-up. Of course, not all patients will recur; the recurrence rate reported in a recent meta-analysis is around $15.2 \%$ (2), ranging from $5 \%$ to $47.4 \%$, depending on the applied criteria for recurrence. Thus, in the era of precision medicine, it would be of particular importance to have predictive markers that can identify patients with a low likelihood of relapse, who may be reassured and subjected to less frequent follow-up, as well as patients with an increased likelihood of relapse, who may be advocated a more intensive follow-up.

Recurrence prediction following TSS is inherently challenging in CD. No accurate predictive biomarkers of long-term outcome have yet been established (3). Although the most widely applied criterion is the measurement of early postoperative levels of cortisol after hydrocortisone discontinuation, accumulating evidence suggests that it may not be as accurate as originally thought. A number of other biomarkers, such as CRH test, late night salivary cortisol, low-dose dexamethasone suppression, or prolonged postoperative corticosteroid dependence, have been evaluated without superior results. In a recent issue of European Journal of Endocrinology, Cambos et al. (4) retrospectively evaluated the predictive value of the desmopressin test (DT) performed during the early

Published by Bioscientifica Ltd. 
postoperative period in 95 patients with $\mathrm{CD}$ who achieved remission post-TSS. The study confirmed previous findings (reviewed in (5)), indicating a predictive value of DT performed in the early postoperative period regarding the long-term outcome following TSS. Even this study, however, does not circumvent as a possible source of bias the typical limitations shared among studies dealing with patients with rare diseases related to the limited number of patients and the retrospective design. Nevertheless, this is the largest study performed so far, originating from the collaboration of two centers dedicated in the treatment of $\mathrm{CD}$ and, despite its retrospective nature, it remains informative, given the difficulties in conducting prospective studies when a long period of follow-up is warranted in order to assure accurate outcome assessment.

The desmopressin test has an evolving role in Cushing's syndrome (5). Administration of desmopressin elicits paradoxical ACTH and subsequently cortisol responses from neoplastic cells that aberrantly express V2 receptors. This is commonly the case not only with corticotroph adenomas but also, rarely, with neoplastic cells with ectopic ACTH secretion (5). This attribute makes DT a useful tool in the diagnosis of Cushing's syndrome, differentiating neoplastic from functional hypercortisolism (5). In this line, its major advantage in the postoperative assessment of patients lies on the concept that persistence of this response indicates residual presence of neoplastic corticotrophs, even in patients with biochemical evidence of remission, providing the ground for its use to predict longterm outcome. For a trustworthy postoperative use of DT, however, it is essential to document a positive response preoperatively. In this study, all patients were deemed positive to the preoperative DT. To this end, the authors used a control group consisting of patients with functional hypercortisolism, non-corticotroph pituitary adenomas, and overweight/obese subjects. This is somehow advantageous from previous studies that applied largely arbitrary criteria (5) and such an approach is highly recommended in order to incorporate the DT in routine clinical practice.

Currently, assessment of DT in the early post-TSS period remains largely empirical. The criteria used when the test is performed in eu- or hyper-cortisolemic subjects cannot be applied when cortisol levels are in the range of adrenal deficiency, which is usually the case during the early postoperative period. Calculation of percentage increases, in the post-TSS setting, is inappropriate since it may erroneously lead to positive results due to the low value of the denominator. Thus, cut-offs using either absolute changes or peak values are more robust. Also, although in most studies both cortisol and ACTH are measured during the test, cortisol thresholds perform better, probably due to the much higher variability of ACTH levels and/or inaccuracies of ACTH assays. In this study, the investigators report that patients with a cortisol peak $<100$ $\mathrm{nmol} / \mathrm{L}(3.6 \mu \mathrm{g} / \mathrm{dL})$ or a cortisol increment of $<30 \mathrm{nmol} / \mathrm{L}$ $(1.1 \mu \mathrm{g} / \mathrm{dL})$ were less likely (odds ratios $0.12,95 \%$ CI 0.03 0.41 and $0.11,95 \%$ CI $0.02-0.36$, respectively) to have $\mathrm{CD}$ recurrence. Previous studies applying similar criteria proposed a much higher value of 193-204 nmol/L (7-7.4 $\mu \mathrm{g} / \mathrm{dL}$ ) for the cortisol increment, a difference that probably results from heterogeneity among the study populations. In particular, Cambos et al. (4) included patients with very low postoperative cortisol levels potentially explaining the lower estimates for the cortisol increment. Taken together, these data demonstrate the lack of a robust criterion in evaluating DT responses in the immediate post-TSS period. Establishing unanimously accepted criteria is unlikely, given that homogeneity is difficult to achieve among studies of $\mathrm{CD}$ and this is not surprising since even in the definitions of remission and recurrence there is still much equivocacy. Thus, each center needs to validate the test in its own patient population and this is a major drawback for its wide routine application.

A secondary, albeit clinically imperative, finding of this study is that it adds to the existing data that low postoperative cortisol levels, apart from being a good marker of immediate remission, do not necessarily imply long-lasting remission $(6,7,8,9)$. Very low or undetectable early postoperative cortisol levels have been traditionally used as a marker of complete corticotroph adenoma removal based on the assumption that the normal pituitary corticotroph cells are completely suppressed by the preceding hypercortisolism (10). In this study, despite the fact that only patients with an early postoperative morning cortisol of $<138 \mathrm{nmol} / \mathrm{L}(5 \mu \mathrm{g} / \mathrm{dL})$ were included, a significant proportion of $18 \%$ did recur within a median of 30 months from surgery, underlining the fact that recurrence constitutes a significant problem, even in patients achieving what is currently regarded as the best possible surgical outcome.

In contrary, it is well-documented that around 10\% of patients with higher postoperative cortisol levels will still undergo long-term remission (11) and that care is needed in order to avoid unnecessary second surgery in this particular group. Postoperative cortisol levels may not be suppressed for many reasons such as mild or cyclic forms of Cushing's disease, short duration, or the use of medical treatment before surgery. Adrenal hyperplasia 
and/or partly autonomous nodules secondary to the chronic ACTH stimulation with residual cortisol secretion that successively subsides may also affect immediate postoperative cortisol levels, invalidating them as early and/or late outcome predictive markers. Of note, limited recent evidence indicate that a negative postoperative DT may be a good marker of remission in these patients, protecting them from unnecessary interventions (7).

In conclusion and in light of the accumulating data showing that basal postoperative levels of cortisol may not represent a reliable marker of the log-term TSS outcome, the DT may evolve as a more powerful prognostic test. The advantage of DT relies on the fact that it targets directly the neoplastic adenoma cells, explaining its better performance compared to baseline cortisol levels which is a rather imprecise measure influenced by many factors $(6,11)$. Clinicians, however, should be aware that it applies only to patients with a documented positive preoperative DT and that only in these patients the disappearance of the paradoxical response is a marker of good prognosis. On the other hand, persistence of DT responsiveness should result in a thorough follow-up and an increased alertness for promptly detecting recurrence. The lack of robust criteria to define positive postoperative responses currently represents a major limitation, and ongoing validation of DT at each dedicated center is required before its wide implementation in the standard of care of surgically treated Cushing's disease patients.

\section{Declaration of interest}

The authors declare that there is no conflict of interest that could be perceived as prejudicing the impartiality of this commentary.

\section{Funding}

This research did not receive any specific grant from any funding agency in the public, commercial, or not-for-profit sector.

\section{References}

1 Clayton RN, Jones PW, Reulen RC, Stewart PM, Hassan-Smith ZK, Ntali G, Karavitaki N, Dekkers OM, Pereira AM, Bolland M et al.
Mortality in patients with Cushing's disease more than 10 years after remission: a multicentre, multinational, retrospective cohort study. Lancet: Diabetes and Endocrinology 20164 569-576. (https://doi. org/10.1016/S2213-8587(16)30005-5)

2 Petersenn S, Beckers A, Ferone D, van der Lely A, Bollerslev J, Boscaro M, Brue T, Bruzzi P, Casanueva FF, Chanson P et al. Therapy of endocrine disease: outcomes in patients with Cushing's disease undergoing transsphenoidal surgery: systematic review assessing criteria used to define remission and recurrence. European Journal of Endocrinology 2015172 R227-R239. (https://doi.org/10.1530/EJE-140883)

3 Nieman LK, Biller BM, Findling JW, Murad MH, Newell-Price J, Savage MO, Tabarin A \& Endocrine Society. Treatment of Cushing's syndrome: an Endocrine Society clinical practice guideline. Journal of Clinical Endocrinology and Metabolism 2015100 2807-2831. (https:// doi.org/10.1210/jc.2015-1818)

4 Cambos S, Mohammedi K, Castinetti F, Saie C, Young J, Chanson PH \& Tabarin A. Persistent cortisol response to desmopressin predicts recurrence of Cushing's disease in patients with post-operative corticotropic insufficiency. European Journal of Endocrinology 2020 182 489-498. (https://doi.org/10.1530/EJE-19-0770)

5 Vassiliadi DA \& Tsagarakis S. DIAGNOSIS of ENDOCRINE DISEASE: The role of the desmopressin test in the diagnosis and follow-up of Cushing's syndrome. European Journal of Endocrinology $2018 \mathbf{1 7 8}$ R201-R214. (https://doi.org/10.1530/EJE-18-0007)

6 Yap LB, Turner HE, Adams CB \& Wass JA. Undetectable postoperative cortisol does not always predict long-term remission in Cushing's disease: a single centre audit. Clinical Endocrinology 200256 25-31. (https://doi.org/10.1046/j.0300-0664.2001.01444.x)

7 Vassiliadi DA, Balomenaki M, Asimakopoulou A, Botoula E, Tzanela M \& Tsagarakis S. The desmopressin test predicts better than basal cortisol the long-term surgical outcome of Cushing's disease. Journal of Clinical Endocrinology and Metabolism 2016101 4878-4885. (https://doi.org/10.1210/jc.2016-2799)

8 Alexandraki KI, Kaltsas GA, Isidori AM, Storr HL, Afshar F, Sabin I, Akker SA, Chew SL, Drake WM, Monson JP et al. Long-term remission and recurrence rates in Cushing's disease: predictive factors in a single-centre study. European Journal of Endocrinology 2013168 639-648. (https://doi.org/10.1530/EJE-12-0921)

9 Roelfsema F, Biermasz NR \& Pereira AM. Clinical factors involved in the recurrence of pituitary adenomas after surgical remission: a structured review and meta-analysis. Pituitary 201215 71-83. (https://doi.org/10.1007/s11102-011-0347-7)

10 Trainer PJ, Lawrie HS, Verhelst J, Howlett TA, Lowe DG, Grossman AB, Savage MO, Afshar F \& Besser GM. Transsphenoidal resection in Cushing's disease: undetectable serum cortisol as the definition of successful treatment. Clinical Endocrinology 199338 73-78. (https://doi.org/10.1111/j.1365-2265.1993.tb00975.x)

11 Valassi E, Biller BM, Swearingen B, Pecori Giraldi F, Losa M, Mortini P, Hayden D, Cavagnini F \& Klibanski A. Delayed remission after transsphenoidal surgery in patients with Cushing's disease. Journal of Clinical Endocrinology and Metabolism 201095 601-610. (https://doi. org/10.1210/jc.2009-1672)

Received 23 March 2020

Accepted 1 April 2020 